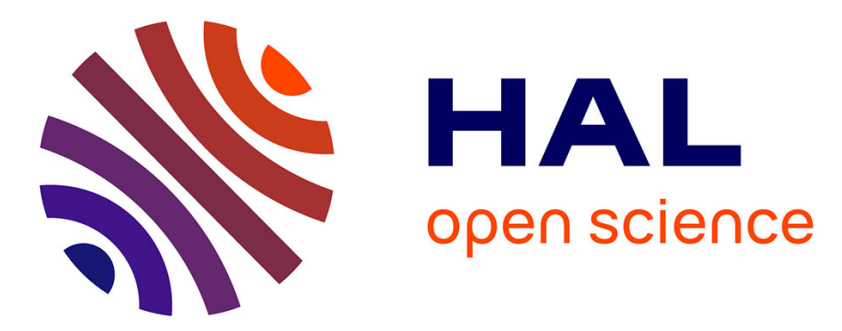

\title{
Unbiased longitudinal brain atlas creation using robust linear registration and log-Euclidean framework for diffeomorphisms
}

Antoine Legouhy, Olivier Commowick, François Rousseau, Christian Barillot

\section{- To cite this version:}

Antoine Legouhy, Olivier Commowick, François Rousseau, Christian Barillot. Unbiased longitudinal brain atlas creation using robust linear registration and log-Euclidean framework for diffeomorphisms. ISBI 2019 - 16th IEEE International Symposium on Biomedical Imaging, Apr 2019, Venise, Italy. pp.1038-1041， 10.1109/ISBI.2019.8759508 . inserm-02099958v2

\section{HAL Id: inserm-02099958 https://www.hal.inserm.fr/inserm-02099958v2}

Submitted on 6 Jun 2019

HAL is a multi-disciplinary open access archive for the deposit and dissemination of scientific research documents, whether they are published or not. The documents may come from teaching and research institutions in France or abroad, or from public or private research centers.
L'archive ouverte pluridisciplinaire HAL, est destinée au dépôt et à la diffusion de documents scientifiques de niveau recherche, publiés ou non, émanant des établissements d'enseignement et de recherche français ou étrangers, des laboratoires publics ou privés. 


\title{
UNBIASED LONGITUDINAL BRAIN ATLAS CREATION USING ROBUST LINEAR REGISTRATION AND LOG-EUCLIDEAN FRAMEWORK FOR DIFFEOMORPHISMS
}

\author{
Antoine Legouhy ${ }^{* \star} \quad$ Olivier Commowick ${ }^{\star} \quad$ François Rousseau $^{\dagger} \quad$ Christian Barillot $^{\star}$ \\ * Univ Rennes, INRIA, CNRS, INSERM, IRISA UMR 6074, Empenn ERL U-1228, F-35000, Rennes, France \\ $\dagger$ IMT Atlantique, LaTIM U1101 INSERM, UBL, Brest, France
}

\begin{abstract}
We present a new method to create a diffeomorphic longitudinal (4D) atlas composed of a set of 3D atlases each representing an average model at a given age. This is achieved by generalizing atlasing methods to produce atlases unbiased with respect to the initial reference up to a rigid transformation and ensuring diffeomorphic deformations thanks to the Baker-Campbell-Hausdorff formula and the log-Euclidean framework for diffeomorphisms. Subjects are additionally weighted using an asymmetric function to closely match specified target ages. Creating a longitudinal atlas also implies dealing with subjects with large brain differences that can lead to registration errors. This is overcome by a robust rigid registration based on polar decomposition. We illustrate these techniques for the creation of a 4D pediatric atlas, showing their ability to create a temporally consistent atlas.
\end{abstract}

Index Terms - Longitudinal atlas, Brain development, Diffeomorphism, Registration

\section{INTRODUCTION}

Brain atlases are a crucial tool in medical imaging. They consist in an unbiased average model of the brain providing a way to compute statistics on populations, understanding brain variability but also to segment regions. Longitudinal (time dependent) atlases allow in addition the comprehension of brain development to highlight changes in growth, shape, structure etc. Those 4-D models are based on a set of 3-D atlases each for a desired timepoint using weight functions that modulate the contributions of the subjects according to their age fit with that timepoint. To produce an unbiased atlas, two different approaches are commonly used. The first one chooses a reference to register all the subjects to and iteratively unbias the atlas with respect to it [1]. The second common approach performs a direct groupwise registration of all the subjects simultaneously [2]. This family of methods however often relies on a number of registrations in the order of the square

* The research leading to these results has been supported by the ANR MAIA project, grant ANR-15-CE23-0009 of the French National Research Agency (http://recherche.imt-atlantique.fr/maia) and La Région Bretagne. of the number of subjects which can quickly be computationally expensive. Atlasing methods such as [1] provide a good average shape and intensity. However they define unbiased atlases up to an affine transformation, thus losing information on brain growth. On the other hand, [3] corrects well for global size and shape (affine features) but ignores local deformations and thus leads to blurry images. [4] presented a technique that takes into account both global and local changes and uses a Gaussian kernel with adaptive width adjusted according to the temporal distribution of the subjects. However, the rigidity of the Gaussian kernel does not guarantee that the age of the resulting atlas corresponds to the expected one.

We propose a longitudinal atlas creation method that is unbiased up to a rigid transformation accounting both for global and local transformations in the unbiasing step. Generalizing the procedure from [1] to longitudinal atlases, it only requires a number of iterations in the order of the number of subjects times the number of iterations. Additionally we extend this framework to handle diffeomorphisms using the log-Euclidean framework [5] and the Baker-CampbellHausdorff formula to quickly compose and average those transformations. In addition, a quintic polynomial weight function is introduced giving enough degrees of freedom to reduce temporal error of the $3 \mathrm{D}$ atlases while ensuring adaptivity to data distribution.

We finally introduce a robust registration method based on polar decomposition to deal with disparities in terms of brain sizes and shapes, especially in the early years (or months) of life. All these contributions are put together to construct a longitudinal atlas from a database of pediatric patients, illustrating the ability of our framework to obtain well defined temporal atlases in a robust manner.

\section{PREREQUISITES}

\subsection{Robust linear registration methods}

A linear transformation is a composition of a linear map $M$ and a translation $t$ operating on coordinates: $y=M x+t$. The transformation type depends upon the constraints on $M$.

Linear registration consists in finding an optimal linear transformation that matches a moving image onto a reference 
image usually by maximizing a similarity criterion. Among those approaches, block matching strategies [6] have gained in popularity for their robustness and simple implementation. After an initialization phase, two steps are iterated: 1matching: for a set of blocks in the reference image, homologous blocks best satisfying a similarity criterion are searched in the moving image, providing a set of paired points $x=\left\{x_{1}, \ldots, x_{k}\right\}$ and $y=\left\{y_{1}, \ldots, y_{k}\right\} ; 2$ - aggregation: an optimization allows to find the global transformation minimizing the distance between the two sets.

The first step is performed in the same way for any linear transformation. The second step is dependant over the type of linear transformation leading to an adapted optimization in each case. However, for any linear transformation, the least squares optimization is still split into two successive parts [7]: 1- the optimal translation is a function of the optimal linear map $\hat{M}$ and of the two sets barycenters $\bar{x}$ and $\bar{y}: \hat{t}=\bar{y}-\hat{M} \bar{x}$ ;2- $M$ is optimized depending on its constraints (e.g. rotation matrix for a rigid transformation).

Direct rigid registration can be corrupted by strong similarities on the edges of the brain. Estimating directly a rigid transformation between the images may then lead to shifted brain barycenters and misaligned mid-sagittal planes. To correct this, we propose to first estimate an affine transformation $\hat{T}_{A}$ and then extract the rigid part from it through polar decomposition (PD). PD allows the factorization of a matrix $M$ into $M=U H$, where $U$ is a unitary matrix and $H$ is a positive-semidefinite Hermitian matrix. By taking $R=\operatorname{Det}(U) \cdot U$ and $S=\operatorname{Det}(U) \cdot H$, we can separate $M$ into a stretching part $S$ that encapsulates scaling along a set of orthogonal directions and a rotation part $R$ excluding reflection. Using PD on $\hat{M}(\hat{M}=\hat{R} \hat{S})$, a robust rigid transformation $\hat{T}_{R}$ can be extracted by using: $\hat{R}$ for the rotation map and $\bar{y}-\hat{R} \bar{x}$ for the translation part. The remaining affine transformation $\hat{T}_{S}$ (so that $\hat{T}_{A}=\hat{T}_{R} \hat{T}_{S}$ ), is then obtained as $\hat{T}_{S}=\hat{T}_{R}^{-1} \hat{T}_{A}$.

\subsection{Log-Euclidean framework on diffeomorphisms and the Baker-Campbell-Hausdorff formula}

Diffeomorphisms have been chosen for non linear registration due to their interesting properties: differentiability, bijectivity and differentiability of the inverse map. However, computation of Euclidean operations on them does not generally yield a diffeomophism nor an invertible transformation at all. This is however a crucial part in atlas creation where the inverse of an average of diffeomorphisms is needed. To cope with this, Arsigny et al. [5] introduced a framework to deal with diffeomorphisms parameterized by Stationary Velocity Fields (SVF) based on their embedding into a pseudo-Lie group. This log-Euclidean framework allows fast computation of average transformations (Euclidean operations on SVFs) while ensuring them to remain diffeomorphisms.

The computation of the SVF resulting from the composition of two diffeomorphisms, i.e. $\log (\exp (v) \circ \exp (w))$ with $v$ and $w$ SVFs, is however very long as it requires the estimation of the principal logarithm. The $\mathrm{BCH}$ formula states that in a $\mathrm{BCH}-\mathrm{Lie}$ group, for $v$ and $w$ small enough, $\log (\exp (v) \circ \exp (w))$ can be approximated as a series of Lie brackets: $\log (\exp (v) \circ \exp (w)) \approx v+w+\frac{1}{2}[v, w]+\ldots$ Where $[v, w](x)=\operatorname{Jac}(v)(x) \cdot w(x)-\operatorname{Jac}(w)(x) \cdot v(x)$. It has been shown in [8, 9] that the use of the $\mathrm{BCH}$ formula is well suited for diffeomorphisms. In particular, knowing only the SVFs of two diffeomorphisms, the SVF of their composition can be approximated without any logarithm computation.

\subsection{Quintic polynomial weight function}

Given a sample of $N$ subjects of ages $\left\{\tau_{1}, \ldots, \tau_{N}\right\}$, to create an atlas representative of an age $\tau$, it seems natural to give more importance to subjects closer in age to $\tau$. This can be done by associating to each subject $i$ a weight $w_{i}$ based on its temporal difference to $\tau$. The weights are then used in the averaging process (see section 3). The most common approach is to use a Gaussian kernel centered in $\tau$ with standard deviation $\sigma$. An adaptative $\sigma$ can be used to harmonize the number of subjects of non-negligible weight in case of non-uniformly distributed sample ages [4]. Still, the rigidity of the Gaussian kernel implies that if the $\tau_{i}$ are not distributed symmetrically around $\tau$, the temporal error $E=\left|\tau-\sum_{i} w_{i} \tau_{i}\right|$ between $\tau$ and the computed age will be large leading to a temporally shifted atlas. We thus propose instead to use a more flexible weight function, namely a quintic polynomial $P$, supported on a window $[\alpha, \alpha+\delta]$ with the following constraints:

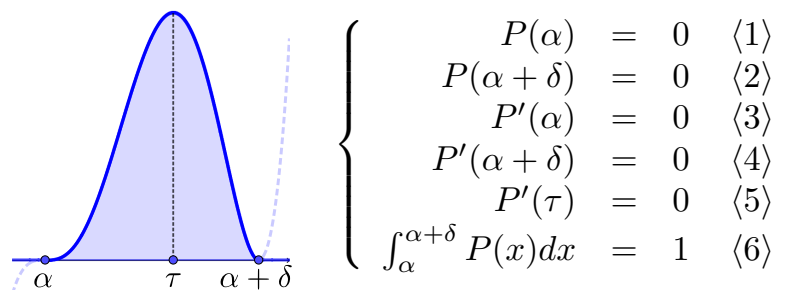

Constraints $\langle 1\rangle$ and $\langle 2\rangle$ implie: $P(x)=(x-\alpha)(x-(\alpha+$ $\delta))\left(a x^{3}+b x^{2}+c x+d\right)$. In turn, this leads to a system of 4 equations (remaining constraints) and 4 unknowns which has an analytic solution. In addition, it is also necessary that $P(x) \geq 0, \quad \forall x \in] \alpha, \alpha+\delta[$ and $\tau \in[\alpha, \alpha+\delta]$. This condition is fulfilled for $\alpha \in[\tau-3 \delta / 5, \tau-2 \delta / 5]$. The flexibility of this weight function allows to optimize over $\alpha$ in its bounds to minimize $E$ for a given $\tau$. The width of the kernel $\delta$ allows to adapt to data disparities to guarantee a number of subjects used to compute the atlas. This is done using an iterative procedure similar to [4]. The age range is subsampled into a collection of potential ages and an ideal number of subjects $n$ per atlas is chosen. Then, for each tentative age, if the number of subjects inside the window is less than $n, \delta$ is increased, otherwise it is reduced. Finally, smoothing is applied for a smooth transition between consecutive potential ages. 


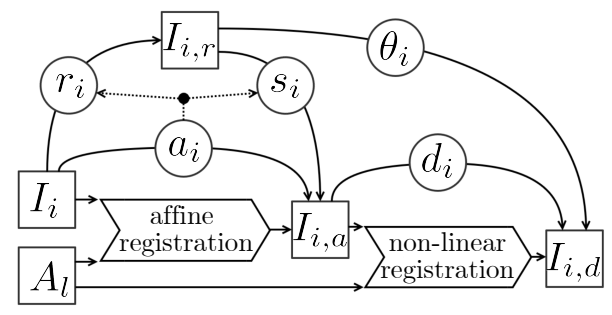

Fig. 1. Two steps registration process of $I_{i}$ on $A_{l}$ : affine registration decomposed in a rigid part $r_{i}$ and a stretching part $s_{i}$ followed by a diffeomorphic registration (transformation $d_{i}$ ).

\section{LONGITUDINAL ATLAS CREATION METHOD}

We define a longitudinal (4D) atlas as a succession of 3D atlases each representing an average model at a given age. Those atlases are created using a generalized version of the method proposed by Guimond et al. [1] to provide an unbiased 3D atlas up to a rigid transformation and with variable weights. For each desired age, an initial reference is chosen among the subjects and the following steps are then iterated:

1. Registration: all the subjects are registered on the current reference image $A_{l}$.

2. Creation of the new reference $A_{l+1}$ : i- computation of $\bar{\theta}$ the weighted average of output transformations, iicomputation of $\bar{I}$ the weighted average of registered images, iii- application of $\bar{\theta}^{-1}$ to $\bar{I}$ to obtain $A_{l+1}$.

This creates a succession of reference images that are less and less biased by the choice of the first reference image and converges to an unbiased average model of the subjects naming an atlas. To be representative of a given time $\tau$, it is necessary to give more importance to subjects with an age closer to $\tau$. This is handled in the averaging processes described below.

Registration. At a given iteration $l$, each subject $i$ is registered onto $A_{l}$ in two steps using our block matching registration implemented in the Anima open source software (see Fig. 17. First, an affine registration is performed using [6]. The algorithm is initialized by a transformation determined using principal component analysis on the non-zero voxels of the two images. This makes the barycenters of the two images coincide and align and stretch their principal directions according to the corresponding eigenvalues. The output transformation is decomposed, using the method depicted in Section 2.1. into a robust rigid part $r_{i}$ and a stretching part $s_{i}$. The second step is a diffeomorphic registration [10] that outputs a SVF $\log \left(d_{i}\right)$. We therefore end up with a series of transformations separated into two parts: $r_{i}$ and $\theta_{i}=s_{i} \circ d_{i}$. To further simplify $\theta_{i}$ and average them in a simple manner, we compute the SVF associated to $s_{i}$ by taking its matrix logarithm and applying it to each spatial point of the image, thus obtaining a SVF $\log \left(s_{i}\right)$ [11]. The logarithm of the composi-

\footnotetext{
${ }^{1}$ Anima: https://goo.gl/HDC5Jf
}

tion of $s_{i}$ and $d_{i}$ is then approximated through the $2^{\text {nd }}$ order $\mathrm{BCH}$ formula (see Section 2.2).

Creation of the new reference image. After the registration stage, we then compute the new average reference for the temporal point of interest from a set of SVFs $\left\{\log \left(\theta_{i}\right)\right\}$ and a set of registered images $\left\{I_{i, d}\right\}$. The inverse of the log-Euclidean average of the transformations (Section 2.2 $\bar{\theta}^{-1}=\exp \left(-\sum_{i} w_{i} \log \left(\theta_{i}\right)\right)$ is then applied to the average of registered images: $\bar{I}=\sum_{i} w_{i} I_{i, d}$ to create $A_{l+1}$. Doing so over several iterations ensures, similarly to Guimond et al., to obtain an unbiased atlas this time with respect to a rigid transformation. To compute the $3 \mathrm{D}$ atlas for a given age, the weights $w_{i}$ are chosen using the weight function depicted in Section 2.3 and normalized such that they sum up to 1.

\section{MATERIAL AND RESULTS}

Our method has been tested on T1 images (size: $200 \times 200 \times$ 200, voxel resolution: $1 \times 1 \times 1 \mathrm{~mm}^{3}$ ) from the C-MIND database, a data repository created for the study of normal brain development conducted by Cincinnati Childrens Hospital Medical Center and UCLA and supported by the National Institute of Child Health and Human Developmen 2 , 197 healthy subjects with ages ranging from less than a month to almost 19 years old have been selected after quality check. Temporal error evaluation. We have chosen the following parameters for the weight functions algorithm: desired number of subjects per 3D atlas: $n=25$, ages subsampling: from 0 to 19 years with a step 0.01 , smoothing using LOESS with window 50. The main advantage of our weight function is the possibility of obtaining an asymmetric function to reduce temporal error through optimization over $\alpha$. We thus first compared our method (a) with a similar one (b) with a fixed $\alpha=T-\delta / 2$ leading to symmetric weight functions around $\tau$. To avoid side effects, calculations have been performed on the interval $\left[\tau_{\min }, \tau_{\max }\right]=[0.76,16.5]$ such that $95 \%$ of subjects ages are greater than $\tau_{\min }$ and $95 \%$ less than $\tau_{\max }$. The temporal errors for each method are shown in Table 1 Method (a) proves its ability to correct the temporal error allowing to construct well-timed atlases up to a day for almost half of the age range while it happens only for $2 \%$ using method (b).

\begin{tabular}{|c|c|c|c|}
\hline Method & Median error & Error $<$ day (\%) & Error $<$ week (\%) \\
\hline $\mathrm{a}$ & 0.0002 & 48.5684 & 56.5748 \\
\hline $\mathrm{b}$ & 0.0686 & 2.0148 & 14.263 \\
\hline
\end{tabular}

Table 1. Comparison of the median of the temporal errors (in years), percentage of timepoints where the error is less than 1 day and 1 week respectively for methods (a) and (b).

Pediatric atlas construction. We then conducted the creation of a pediatric atlas from the C-MIND database. The parameters were set as: number of iterations: 8 , atlas timepoints com-

\footnotetext{
${ }^{2}$ C-MIND: https: //research. cchmc.org/c-mind
} 


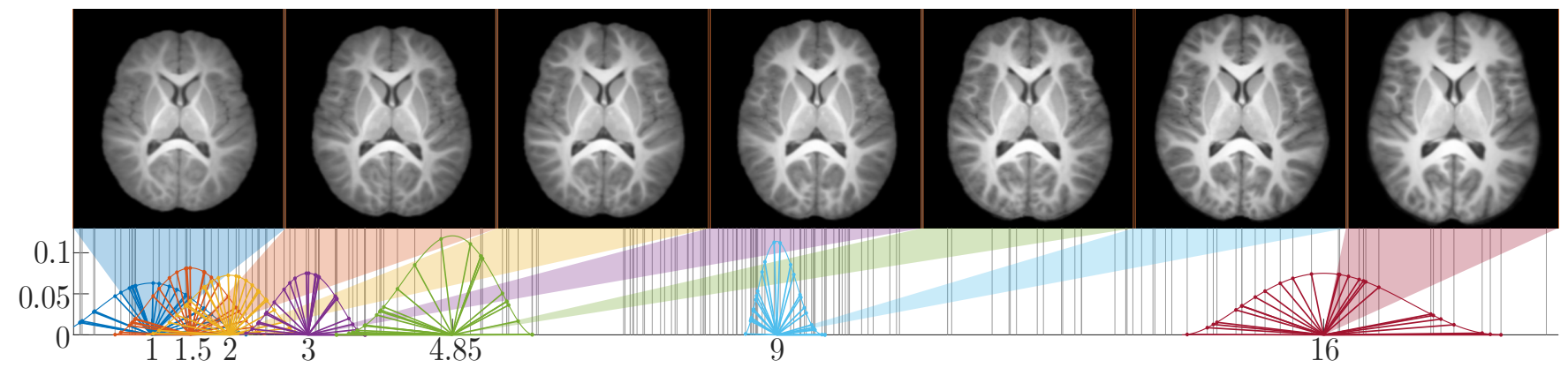

Fig. 2. Longitudinal atlas (top) and associated weight functions (bottom) for ages: 1, 1.5, 2, 3, 4.85, 9 and 16 years. Vertical lines represent ages of C-MIND subjects.

puted: $1,1.5,2,3,4.85,9$ and 16 years old (ages for which the temporal error was less than a day). Our method provides atlases for each age that correspond to the subjects growth apart from a rigid transformation. We have therefore used the oldest subject with a non-zero weight for each atlas timepoint as the first reference. For visualization purposes, we have additionally registered robustly with a nearest rigid transformation (see Section 2.1) each of these initial references on an external atlas so that all temporal atlases are rigidly aligned. We present the computed atlases in Fig. 2 together with the subjects ages used for their construction.

Visual checkup on this longitudinal atlas explicits quick general brain growth in the early stages of life until a stabilization around 5 years old except for the antero-posterior direction that continues until the older ages. Thanks to our robust rigid registration, all temporal atlases are well centered on their barycenters and their sagittal planes are well aligned.

\section{CONCLUSION}

We presented a longitudinal atlas creation method using a generalized version of the algorithm from [1]. It takes advantages of the log-Euclidean framework and the $\mathrm{BCH}$ formula to produce individual age atlases unbiased up to a rigid transformation giving the opportunity to highlight global changes and local deformations in the atlas. To be representative of a given age, we introduced a quintic polynomial weight function to modulate the influence of each subject. Through its flexibilty (potential asymmetry), it ensures a large choice of ages on which the associated atlas is not temporally erroneous. Finally, by denoting $N$ the number of subjects and $L$ the number of iterations in the main loop of the algorithm, our method necessitates only a number of registrations in $\mathcal{O}(M N)(M$ being in practice much smaller than $N$ ) while numerous atlas creation methods require this number to be in $\mathcal{O}\left(N^{2}\right)$.

\section{REFERENCES}

[1] Alexandre Guimond, Jean Meunier, and Jean-Philippe Thirion, "Average brain models: A convergence study,"
CVIU, vol. 77, no. 2, pp. 192-210, 2000.

[2] T. Rohlfing, N. M. Zahr, et al., "The SRI24 multichannel atlas of normal adult human brain structure," HBM, vol. 31, no. 5, pp. 798-819, 2009.

[3] Maria Kuklisova-Murgasova, Paul Aljabar, et al., "A dynamic 4D probabilistic atlas of the developing brain," NeuroImage, vol. 54, no. 4, pp. 2750-63, 2011.

[4] Ahmed Serag, Paul Aljabar, et al., "Construction of a consistent high-definition spatio-temporal atlas of the developing brain using adaptive kernel regression," NeuroImage, vol. 59, no. 3, pp. 2255-65, 2012.

[5] V. Arsigny, O. Commowick, et al., "A Log-Euclidean Framework for Statistics on Diffeomorphisms," in MICCAI, 2006, vol. 4190 of LNCS, pp. 924-931.

[6] O. Commowick, N. Wiest-Daesslé, and S. Prima, "Block-matching strategies for rigid registration of multimodal medical images," in ISBI, 2012, pp. 700-703.

[7] Berthold K. P. Horn, "Closed-form solution of absolute orientation using unit quaternions," Journal of the Optical Society of America A, vol. 4, no. 4, pp. 629, 1987.

[8] Matias Bossa, Monica Hernandez, and Salvador Olmos, "Contributions to 3D Diffeomorphic Atlas Estimation: Application to Brain Images," in MICCAI, 2007, vol. 4791 of $L N C S$, pp. 667-74.

[9] T. Vercauteren et al., "Symmetric Log-Domain Diffeomorphic Registration: A Demons-Based Approach," in MICCAI, 2008, vol. 5241 of LNCS, pp. 754-761.

[10] O. Commowick, N. Wiest-Daesslé, and S. Prima, “Automated diffeomorphic registration of anatomical structures with rigid parts: application to dynamic cervical MRI," in MICCAI, 2012, pp. 163-70.

[11] V. Arsigny, O. Commowick, et al., "A fast and logeuclidean polyaffine framework for locally linear registration," JMIV, vol. 33, no. 2, pp. 222-238, 2009. 